\title{
Gestión universitaria y del conocimiento aplicada a la capacitación de su PASyT. (personal administrativo, de servicios y técnico) con inclusión de las TICs
}

\author{
Ana María Trottini \\ Juan Emilio Contesti \\ (Universidad Nacional de Rosario. Argentina) \\ (Universidad Abierta Interamericana. Argentina)
}

\section{Resumen}

Para comprender las transformaciones que vienen produciendo las tecnologías digitales en las sociedades se necesita recurrir a un análisis profundo de los modos en que el hombre interactúa con otros hombres. Pero lo que lo diferencia de otros es que ahora el conocimiento actúa sobre sí mismo generando más conocimiento.

A medida que se genera un nuevo conocimiento y se desarrollan nuevas habilidades, el gestor debe perfeccionar sus saberes, la capacitación debe ser continua.

Necesitamos desarrollar la capacidad de asociación, de saber qué tipo de cualificaciones resultan imprescindibles para las tareas que tenemos que hacer, dónde buscarlas, cómo aprenderlas y cómo incorporarlas al mundo laboral.

El propósito de este análisis es ubicar a la Universidad en el área de la capacitación continua de su capital humano, y proponer caminos alternativos para que pueda seguir cumpliendo con su misión, ya que una gestión inteligente contribuye al funcionamiento más dinámico.

Cualquier organización que aspire al éxito deberá tener colaboradores con altos parámetros asociados a la flexibilidad, a la comunicación interactiva, a la apertura mental, a un comportamiento de asociación comprometida entre pares.

Este estudio brinda respuestas a la necesidad de capacitación continua del personal universitario, desde la Gestión.

\section{Palabras clave}

Gestión del conocimiento - Tecnologías digitales - Capital Humano - Organización Universidad.

\section{Summary}

An intense epistemological debate becomes strong in the eighties in the field of the knowledge and education management, and there is consolidated the need to 
revalue the criteria of efficiency and effectiveness in the management of government of Organizations of High Education and the value of the TICs in his dynamic.

To understand the transformations that produce the digital technologies in the societies we need to resort to the analysis of the manners in which the man interacts with other men. But the notable thing is that now the knowledge acts over itself, generating more knowledge. We investigate the knowledge management, the but there are no sufficient studies on the personnel management in South America.

On new knowledge having generated and setting up new skills, the manager must perfect what he has learned with constant training, develop the capacity of association, to know what kind of skills turn out to be indispensable for the assigned tasks, where to look for them, how to learn them and how to incorporate them into the labour world.

This analysis seeks to locate the University, from the continue training of his human capital, and propose alternatives to continue with his Mission, because an intelligent management, contributes to a more dynamic functioning.

Any organization that aspires to success will have to have collaborators with parameters associated with the flexibility, the interactive communication, the mental opening, and also to a solidarity behavior compromised between couples.

This study provides answers to the need of constant training of the university personnel, from the Management.

\section{Key words}

Knowledge management - Digital Technologies - Human Capital - Organization University.

\section{Introducción}

La creciente complejidad de los sistemas educativos y de la teoría organizacional y administrativa lleva a una permanente reconstrucción del conocimiento. La mente humana es una fuerza productiva directa, no sólo un elemento decisivo del sistema de producción: las computadoras, los sistemas de comunicación, y la decodificación y programación genética son todos amplificadores y extensiones de la mente humana.

Las teorías de la organización y de la gestión educativa han permitido examinar el papel mediador de la administración en la gestión. Gestión que se elabora en un contexto de interdependencia internacional, desarrollo humano sostenible y gerencia social. La administración moderna está concebida como un instrumento para organizar y coordinar la prestación de los servicios. Compete a la gestión educativa dar soluciones específicas a los problemas reales que se presentan en sus organismos.

Se hace imprescindible la incorporación de técnicas de planificación estratégica y calidad total en la administración de la Educación Superior, porque la planificación estratégica nos permite tomar decisiones, teniendo en cuenta la adaptación de los recursos y habilidades de la organización, a las exigencias 
del cambiante contexto en el que estamos inmersos, aprovechando las oportunidades y evaluando los riesgos, en función de los objetivos de la institución.

La introducción de estas tecnologías implica un cambio en profundidad de la propia sociedad. Las nuevas tecnologías de la información y la comunicación (TICs) designan a la vez un conjunto de innovaciones tecnológicas pero también las herramientas que permiten una redefinición radical del funcionamiento de la sociedad. La puesta en práctica de las TICs afecta a numerosos ámbitos de las ciencias humanas, la teoría de las organizaciones y la gestión.

Nuestra investigación se ha nutrido en el paradigma de la Complejidad (Morin, 2000), tomando en consideración los tres principios en los que se inspira metodológicamente: el principio dialógico; el recursivo organizacional y el hologramático a fin de poder analizar la Universidad como organización y la gestión del capital humano.

\section{La organización universidad y la gestión del capital humano}

En una institución tan compleja como la universitaria, resulta, hoy día, imprescindible el cambio organizacional para lograr mejor calidad y eficiente servicio.

Tomando en cuenta las culturas organizacionales vigentes y los nuevos contextos podemos identificar algunos objetivos fundamentales de la planificación tales como:

- Fortalecer la capacidad de gestión estratégica, abandonando las prácticas sectoriales y corporativas.

- Jerarquizar las funciones directivas y de gestión apelando en lo posible a la formación específica en postgrados.

- Crear nexos permanentes entre las prácticas de gestión, el diseño de políticas de conocimiento y de información, los cambios y reformas curriculares, los programas de evaluación y las reformas institucionales.

Ante estos objetivos un equipo de gestión debería suplir las competencias que un individuo solo no puede cubrir.

\section{Gestión del P.A.S. y T., descripción, funciones, responsabilidades}

En los debates de la Conferencia Mundial sobre Educación Superior, auspiciada por la UNESCO (París 1998), se reconocieron cambios esenciales para la Educación Superior, en la perspectiva de una educación a lo largo de toda la vida, además de reconocer que estos servicios deben tener un carácter masivo y no elitista.

Los esfuerzos por contribuir al perfeccionamiento de la Educación Superior y superar el estado general de crisis que la misma presenta a nivel mundial, tienen como un hito trascendental la Conferencia Mundial antes mencionada, la cual fue precedida por conferencias regionales en La Habana (noviembre, 
1996), Dakar (abril, 1997), Tokio (julio, 1997), Palermo (septiembre, 1997) y Beirut (marzo, 1998); en las que se reflexionó profundamente sobre los problemas y retos existentes y en las que tuvo un importante espacio el análisis de la gestión universitaria, dado que fue uno de los temas centrales abordados, junto con la pertinencia, la calidad, la acreditación y la cooperación internacional.

Así en la Declaración Mundial sobre Educación Superior en el Siglo XXI, se expresa: "La educación superior ha dado sobradas pruebas de su viabilidad a lo largo de los siglos y de su capacidad para transformarse y propiciar el cambio y el progreso de la sociedad. Dado el alcance y el ritmo de las transformaciones, la sociedad cada vez tiende más a fundarse en el conocimiento, en razón de que la educación superior y la investigación forman hoy día parte fundamental del desarrollo cultural, socioeconómico y ecológicamente sostenible de los individuos, las comunidades y las naciones. Por consiguiente, y dado que tiene que hacer frente a imponentes desafíos, la educación superior ha de emprender la transformación y la renovación más radicales que jamás haya tenido por delante, de forma que la sociedad contemporánea, que en la actualidad vive una profunda crisis de valores, pueda trascender las consideraciones meramente económicas y asumir dimensiones de moralidad y espiritualidad más arraigadas" (UNESCO, 1998).

Hoy día, la Declaración de la Conferencia Regional de Educación Superior en América Latina y el Caribe -CRES 2008-, apunta a la integración regional y a la internacionalización de los sistemas nacionales de educación superior (SNES) como uno de los elementos estratégicos para enfrentar los retos y oportunidades que se plantean en la educación superior de la región, reafirma la importancia del "... mutuo conocimiento entre los sistemas como base para la movilidad académica y como insumo para adecuadas políticas públicas e institucionales" y propone "la articulación de los sistemas nacionales de información sobre Educación Superior de la región, a través del Mapa de la Educación Superior en América Latina y el Caribe (MESALC)". Los desafíos hacia una Educación Superior de calidad, equitativa y pertinente -expresa José Renato Carvalho- "son de tal magnitud que, de no ser atendidos con oportunidad y eficacia, ahondarán las diferencias, desigualdades y contradicciones que hoy impiden el crecimiento de América Latina y el Caribe con equidad, justicia, sustentabilidad y democracia para la mayoría de los países que la conforman", según destaca la Declaración de la CRES 2008. La premisa fundamental del MESALC es la cooperación regional como el camino más eficiente y eficaz para superar nuestras deficiencias. A través de una tecnología propia, abierta a la colaboración y participación de todos, las organizaciones interesadas consideran si quieren añadir sus contribuciones.

Al analizar el papel de la administración, "si entendemos a la administración como una ciencia que estudia las organizaciones con fines descriptos para comprender su funcionamiento, su relación, su crecimiento y su conducta, resulta evidente que ella tendrá que actuar en la universidad considerando: 
1) Control del sistema que administra (control) y

2) Los propósitos de las personas que forman parte del sistema administrativo (humanización) en la que coexisten grupos con diferentes intereses" (González, 1995). Para el mejoramiento de la calidad de las prácticas educativas, Frigerio y Poggi (1994) adoptan un concepto comprensivo de calidad de educación, definiéndola: "como la integración de la calidad de la organización institucional, de los recursos humanos y financieros, de la conducción educativa, de la propuesta curricular y didáctica, del proceso educativo y de sus resultados en términos de aprendizajes".

La calidad del personal de las universidades ocupa un lugar central, en relación a que la calidad del quehacer educativo está indefectiblemente relacionada con la calidad de los trabajadores de la educación. "Todo esto sin dejar de lado las vivencias del cuerpo administrativo, que si bien carece en la mayoría de los casos de poder explícito, no deja de tener peso en las decisiones que instrumentan y en su elaboración" (González, 1995).

El reto para los individuos que se desarrollan en todas las áreas de conocimiento es vivir de acuerdo a las exigencias de este nuevo tipo de sociedad, estar informados y actualizados, innovar, pero sobre todo generar propuestas y generar conocimiento.

Las sociedades del conocimiento no se reducen a la información, ésta es efectivamente un instrumento del conocimiento, pero no es el conocimiento en sí.

La cognición distribuida ha fomentado un modelo de relaciones sociales basado en la colaboración colectiva. Manuel Castells (2001) considera que "hay una serie de cualidades necesarias para poder ejercer las destrezas que exige hoy el mundo del trabajo. Las más importantes son la flexibilidad, la movilidad y la disponibilidad para aprender rápido y durante toda la vida".

Para impulsar estos cambios se torna decisivo el rol de la gestión, entendida como: la capacidad del gobierno y de la administración de la institución para alcanzar los objetivos propuestos.

Un objetivo es lograr la valoración adecuada del personal y su integración a la institución creándole un sentido importante de pertenencia. Segrelles Cucalas (2000) explica el Proyecto Personal de Vida que establece términos de negociación y compromiso para con la institución. Si bien está planteado para el personal académico, nada impide que se lo extienda al personal de investigación o al administrativo y técnico. Esta planificación individual permite buscar un estímulo al desarrollo personal y al Profesionalismo que se puede alcanzar (Padrino, 2005).

Este compromiso lleva a reconocer los activos intangibles que tienen su origen en los conocimientos, habilidades, valores y actitudes de las personas, a estos activos intangibles se les denomina capital intelectual, o que denominamos "Capital Humano". "Se consideran activos intangibles las capacidades que se 
generan en la organización, cuando los recursos (capital humano) empiezan a trabajar en grupo" (Di Doménico y De Bona, 2005).

La gestión del conocimiento es, en definitiva, la gestión de los activos intangibles que generan valor para la organización y están direccionados hacia ella.

"En esta nueva economía, el recurso por excelencia ya no son los factores económicos tradicionales (tierra, trabajo y capital) sino el conocimiento, un activo que a pesar de no lucir en la contabilidad (por ser intangible) contribuye de forma notable a los resultados de la organización" (1). Para que los individuos generen conocimiento y éste sea integrado y transferido hasta convertirse en conocimiento organizativo, la organización debe proporcionar un contexto adecuado. El profesor Ikujiro Nonaka señala que la conversión del conocimiento se origina mediante la interacción entre conocimiento tácito y explícito durante los procesos de socialización (conversión de tácito a tácito), internalización (conversión de explícito a tácito), externalización (conversión de tácito a explícito) y combinación (conversión de explícito a explícito).

El Capital Intelectual es, por tanto, la variable utilizada para medir el conocimiento organizativo y está formado por capital humano (valor base, valor de relaciones y valor potencial) y capital estructural, que es todo aquello que queda en la organización cuando los empleados se van a casa, es decir, sistemas de información, bases de datos, software de tecnologías de la información (Di Doménico y De Bona, 2005, p. 80).

"Ahora más que nunca la clave del éxito y la excelencia organizacional se centra en las personas y su gestión. Las Instituciones de Educación Superior deberán darse cuenta de que, más allá de las tecnologías y de los procesos, son los conocimientos y el saber de su personal, cada vez más preparados, los que aportan el valor agregado a la organización educacional" (Di Doménico y de Bona, 2005, p. 85).

Viedma Martí (2002) manifiesta que: "los conocimientos que generan valor o el Capital Intelectual son la clave del éxito de las organizaciones que compiten en el contexto económico, político, social y tecnológico de nuestro tiempo, y su papel en el futuro será con toda seguridad cada vez más relevante".

\section{Factores críticos en la gestión del capital humano}

Si se apuesta por eficiencia, eficacia y responsabilidad en la toma de decisiones, es imprescindible apostar por equipos que sepan realizar su gestión con agilidad, eficacia y responsabilidad.

Hay que pensar en todo un período de cambio de los antiguos administradores, a los nuevos profesionistas de la gestión de gobierno de la educación. 
El cambio, en este caso, es un proceso lento y es natural encontrar resistencia al mismo. Hay que contar con gestores educativos que estén capacitados para sortear este problema. Que sean conscientes que están ante personas que no conocen los logros de la administración estratégica y algunos, a poco tiempo de jubilarse, no logran una actitud positiva para internalizar los nuevos conocimientos; y su accionar es un tanto hostil ante el cambio propuesto.

Se deberá trabajar con lo "posible" y no proponerse lograr lo "imposible", no hay que olvidar lo valioso en cuanto a experiencias de trabajo que atesoran estas personas y que las nuevas generaciones deben aprender. Esto no lo enseña una máquina, surge de la buena relación entre personas, que gestionan sus conocimientos entre sí.

\section{¿Qué se está haciendo? ¿Qué se debe mejorar? ¿Cómo hacerlo?}

Las Instituciones de Educación Superior necesitan incorporar, cada vez más, técnicas de planificación y gestión estratégica para elevar la eficiencia y la efectividad de su desempeño, por ende los responsables de la gestión de gobierno deben conocer, capacitarse y ejecutar tecnologías organizacionales tales como:

- Calidad total (Total Quality Management).

- Planificación Estratégica (Strategic Planning).

- Gerencia por objetivos (Management by objetives).

Técnicas que, si bien surgen del ámbito empresarial, se adecuan perfectamente a las universidades sin que peligre su misión y objetivo. Pero, reitero, para que esto sea posible, debe prepararse el capital humano que sepa aplicarlas sin que la institución pierda su esencia.

"La profesionalidad y el saber experto, constituyen condiciones indispensables para asegurar prácticas responsables, éticas y cuidadosas hacia las instituciones y los actores. La profesionalidad no sólo exige instrumentos teóricos y técnicos, sino además una posición ética y una dimensión valorativa de quien toma a su cargo el diseño de este dispositivo" (Frigerio y Poggi, 1994, p. 41). La fundamentación de la necesidad de la Capacitación Formal del PASyT debe realizarse "entendiendo a la capacitación como un proceso sistemático dirigido al mejoramiento de la calidad del capital humano, haciendo uso de todos los medios que conduzcan al incremento del conocimiento, el desarrollo de habilidades y al cambio de actitudes". La capacitación, entonces, implica un desafío que debe responder a las necesidades cambiantes y crecientes de la realidad, fruto del avance acelerado del conocimiento y contribuyendo a la superación.

Augusto Pérez Lindo (2003) plantea que debemos planificar e imaginar una gestión inteligente de las instituciones universitarias.

Luego de la investigación realizada, es evidente que los actores universitarios, profesionales como profesionistas, autoridades como integrantes del 
PASyT, quieren producir este cambio y tienen claro que sólo lo lograrán a través de la capacitación.

Comprobamos que el tema de la Capacitación Continua, propiciado por UNESCO y asentado como Derecho en la Constitución Nacional Argentina, avanza por sobre los intereses particulares y por un camino sin retorno, aunando la sociedad inteligente y la gestión del conocimiento.

Es inobjetable que "la efectividad de una universidad depende esencialmente de la eficiencia y calidad de su personal" (Sanyal, 1995).

Un modelo valioso deberá contemplar como ejes básicos la capacitación, el desarrollo de su potencial, la evaluación y el incentivo, la capacitación permanente en nuevas tecnologías, así como la realización de cursos que permitan cubrir las necesidades de formación de todo el capital humano (Padrino, 2005, p. 168).

La creación de conocimientos en redes y la aceleración del tratamiento de la información abren nuevas posibilidades de trabajo sobre las bases de datos, independientemente de su tamaño, uso y finalidad.

Sus principales aportaciones a las actividades humanas se concretan en una serie de funciones que nos facilitan la realización de nuestros trabajos porque, sean éstos los que sean, siempre requieren una cierta información para realizarlo, un determinado proceso de datos y a menudo también la comunicación con otras personas; y esto es precisamente lo que nos ofrecen las TICs.

Nuestra hipótesis: "La necesidad de capacitación del PASyT, y su realización como capital humano, redunda en beneficio de la calidad del servicio, y la eficiencia desde las universidades hacia la sociedad toda".

Diversos autores coinciden con la necesidad de que la Universidad otorgue a la gestión de capital humano un carácter prioritario, con reconocimiento institucional, que ofrezca un tratamiento global a las problemáticas planteadas.

Este tratamiento debe ser de carácter estratégico, que se consolide en el tiempo, más allá del cambio de gestiones, para garantizar un clima de trabajo confiable, previsible, seguro, que atenúe conflictos, desconfianzas y anticompromiso, propendiendo a la integración de la comunidad, ya que las políticas de capital humano son de la Institución y no deben ser propiedad de la Gestión.

Evidenciamos, entonces, la necesidad de un cambio de la cultura organizacional que saque a la Institución de lo rutinario de la tarea, de la rigidez y del estancamiento. Para que la Institución se adecue a la realidad actual debemos apostar al trabajo en equipo, a la participación, a la solidaridad, al consenso y al compromiso como valores a ser tomados en cuenta para guiar el cambio hacia una nueva gestión que contenga y comprometa a todos los miembros de la comunidad universitaria.

Desde esta perspectiva la capacitación adquiere otra dimensión, dirigiéndola hacia el cambio de actitud, cambio de conducta, motivación, integración, 
desarrollo personal, mejora continua y resolución de conflictos, lo cual evidencia la necesidad de una actitud pro-activa, que asuma las falencias y se comprometa con el cambio.

La solución de los problemas, solo será factible con la participación y el compromiso de todos y cada uno de los integrantes de la comunidad universitaria.

\section{Consideraciones sobre una realidad que nos afecta}

Tendremos que construir una cultura de la gestión pública cuya primera característica básica debe ser la capacitación de todas las personas, en todos los niveles operativos, para cooperar, y esto dependerá en gran parte de la capacidad de comunicarse abiertamente, con libertad y franqueza. Si se quiere lograr que un nuevo modelo funcione, las personas deben ser entrenadas para cooperar en el nivel operativo, sin tener que esperar instrucciones desde niveles superiores. Para que el cambio sea exitoso debe producirse una reforma fundamental ya que una reforma incremental no podría responder a la complejidad de los procedimientos.

Lo positivo radica en contar con una buena estrategia para el cambio.

\section{Nuevo diseño laboral}

Hoy una nueva instancia de diseño laboral se está implementando en las Universidades Nacionales de la República Argentina. Fechado en Buenos Aires el 31 de marzo del 2006, como Decreto 366/2006, entra en vigencia el 1 de mayo del mismo año el nuevo régimen laboral que modifica el número de categorías de 11 a 7, considerando las número 1, 2 y 3 como Tramo Mayor, las 4 y 5 como Tramo Intermedio, y las 6 y 7 como Tramo Inicial.

Es de destacar, en el marco de este trabajo, lo mencionado por parte de los firmantes, como fines compartidos al expresar que "ante la necesidad de adecuarse a los cambios que se vienen produciendo en las Instituciones universitarias nacionales, las partes manifiestan su convicción de acordar y consensuar la implementación de acciones coherentes para encontrar soluciones técnicas y profesionales acordes. Convienen organizar las actividades de acuerdo a las nuevas tecnologías, técnicas y equipamientos, que permitan hacer más productivas las tareas y funciones del personal no docente, utilizando la capacitación, los conocimientos y las habilidades de cada uno y del conjunto de los trabajadores no docentes, los que a su vez se prestarán a la capacitación en su actividad actual o la que potencialmente resulte de sus nuevas habilidades, para el mejor aprovechamiento de las nuevas formas de relación acordadas, y con especial atención a los objetivos institucionales".

El presente análisis iniciado a nivel teórico y luego probado empíricamente nos ha dejado en claro que la Gestión Universitaria es administrar el capital 
humano, y que puede ser una función decisiva para la renovación de nuestras instituciones y para definir las políticas de conocimiento al servicio del desarrollo en América del Sur. Si coincidimos en eso también deberíamos avanzar en la consolidación de una teoría de la gestión universitaria. Esto nos obliga a pensar cómo se articulan los aspectos epistemológicos, administrativos, políticos, institucionales, económicos y pedagógicos de la universidad en un contexto de globalización y desequilibrio (Pérez Lindo, 2005, p. 197).

El permanente desarrollo llevó a la universidad como organización, a requerir una división del trabajo en tareas definidas y la coordinación entre ellas, lo cual generó una nueva actividad que es intrínseca a la organización, la gestión, que a su vez hizo que creara su propia estructura.

La gestión universitaria, lejos de ser uno más de los posibles atributos de un buen rector o una herramienta en el currículo de la administración, se ha tornado una actividad estratégica para el futuro de las instituciones de Educación Superior.

Hemos tratado de analizar la especificidad de la Institución Universidad, para apropiarnos del conocimiento de su complejidad en cuanto a los claustros que la integran y la particular forma de gobierno que la conduce; para luego tomar en especial el análisis de uno de sus claustros, sobre el que menos se ha trabajado, a nivel de especialistas que es el del PASyT, y comparar la diferente y variada metodología que aplica cada Universidad a su capacitación; con el fin de demostrar las similitudes, incoherencias y diferencias entre unas y otras, y concluir con la hipótesis planteada, que corroboro, al manifestar que la universidad tiene una gran deuda con su PASyT que es la de su capacitación sistemática y permanente (UNESCO) la que redundaría en beneficio de la propia universidad, sus servicios, y su hacer social, nacional e internacional.

La reforma debe ser sistemática y metodológica, y tener el consenso del claustro, ya que esto posibilitará su implementación, no sólo para llevarla adelante, con el fin de que el trabajador se adapte a los cambios propuestos, sino también para convertir la reforma en un instrumento de motivación. Reforma que deberá ser flexible, estar expuesta a cambios, ya que una de las virtudes de la misma será precisamente el hecho de no ser definitiva.

EI PASyT debe participar con su aporte de ideas para enriquecer estos principios e ideales, de tal modo que las modificaciones sean construidas por muchos, incorporando la mayor cantidad de cerebros posibles.

Recalcamos la importancia que tiene la reforma académica en la estructura técnico-administrativa, ya que las actividades administrativas y técnicas, como así también las de mantenimiento y servicios generales, están íntimamente relacionadas con las actividades académicas que en el ámbito universitario se desarrollan, y que hoy abarcan necesariamente a la sociedad que las contiene y toma un rasgo, también, internacional.

La reforma organizacional también debe abarcar la estructura de la planta política (autoridades superiores) que, en el caso de las Universidades, ha tenido 
un crecimiento desmesurado sin que esto se tradujera en un funcionamiento más eficaz, más aún, los requerimientos de personal que esto generó, introdujeron más factores de distorsión, sin que se lograra una mayor eficiencia.

La Universidad como organización social no sólo debe contener al PASyT, con fines económicos, sino que debe construir los canales necesarios para que este claustro, muestre su esfuerzo, su saber-saber y también su saber-hacer, que sea conocido y reconocido por los superiores.

Hay principios fundamentales para la reforma que son los de brindar las posibilidades para acceder a:

- Las categorías escalafonarias y sus funciones, y

- La capacitación profesional.

La reforma debe prever nuevos modos de trabajar y diferentes formas de organización laboral, una mayor profundización en materia de capacitación, reformulando los cursos y creando otros nuevos que sean direccionales y más específicos. Las Universidades Nacionales deben promover las medidas necesarias para desarrollar una política eficaz de comunicaciones con el PASyT y su organización laboral, y posibilitarles participar en equipos de trabajo, ámbito que requiere voluntad para enfrentar los problemas y compromiso para solucionarlos, por ello el acceso a la capacitación no debe ser compulsivo. Es muy diferente escuchar decir: "Debo asistir a la capacitación", a "Quiero asistir a la capacitación".

La irrupción de los cursos de capacitación en el sistema universitario nacional nos está indicando que algunas universidades ya han comprendido y aceptado la necesidad de la capacitación pero, no han tomado planificadamente la responsabilidad de esta tarea.

Debe haber capacitación permanente. Y esto es así, ya que la gestión universitaria y sobre todo el propio PASyT, se ve cada vez más agobiado por las crecientes demandas, surgidas en las exigencias de la calidad del servicio, de la planificación y de la toma de decisiones.

La necesidad de capacitación se puede evidenciar por la actitud del PASyT y por los inconvenientes que encuentra en la ejecución de las tareas diarias.

Las corrientes actuales en ciencias de gestión intentan lograr una síntesis de los enfoques psicosociológicos, técnicos y estratégicos con respecto a la organización, que muestran interrelaciones entre el medio, la estrategia, la estructura aplicada, la cultura interna y el comportamiento de los actores.

La universidad tenderá a lograr trabajadores eficientes y que además tengan una formación profesional durante su vida laboral, esto lleva a lograr un eficaz desempeño del PASyT en su puesto o función actual, un aumento en la productividad y los resultados y asegura a la Universidad contar con personal capacitado para cubrir los nuevos puestos originados por la evolución tecnológica, por la creación de nuevas áreas de trabajo y por jubilaciones o retiros. 
Establecida la necesidad de capacitar al PASyT y fijados los objetivos, corresponde planificar las decisiones políticas que se adoptarán para llevar a cabo la capacitación.

El dictado de los cursos de formación, perfeccionamiento y actualización debería estar a cargo de un Centro de Capacitación en Capital Humano, que se financie, a través de la universidad, que podría incluir esta inversión en su presupuesto con un monto no inferior al $1 \%$ que se obtendría del presupuesto asignado al claustro.

En este marco los cursos se realizarán fuera del horario de trabajo, limitándose la institución a brindar las posibilidades y facilidades al trabajador para su concurrencia.

La política de capacitación debe determinar el ámbito y el alcance de los cursos, es decir, el sector y la función del personal que, en una primera etapa, al ser estos cursos direccionados, deberían tener como objetivo principal la nivelación de los conocimientos del PASyT, para así implementar en una segunda etapa, el dictado de Cursos Intensivos, Talleres o Seminarios dentro y fuera de la Universidad, mediante becas o pasantías en otras universidades o Instituciones de Educación Superior (como sucede con el claustro Docente). Estos cursos deben ser de alto nivel.

Una política de capacitación responsable debe involucrar a todos los niveles escalafonarios, por etapas diferentes y agrupamientos, desde el director hasta el empleado ingresante o de diferente jerarquía presupuestaria. De ser posible la responsabilidad de la capacitación debe ser compartida con los propios trabajadores, bajo la dirección de un docente que a la vez sea integrante del PASyT.

Con respecto a las jefaturas y las direcciones, es conveniente que la especialización se lleve a cabo en instituciones y con docentes con nivel de excelencia. Los programas de capacitación tendrán como exigencia primordial que quienes ocupen los niveles de supervisión y jefatura, deban aprender el trabajo que realizan los empleados a su cargo, como un paso previo, para poder capacitar a los niveles inferiores.

En una tercera instancia el plan de carrera deberá ser un desafío productivo y para la universidad debe ser la respuesta laboral que ésta proporciona a la demanda de sus trabajadores.

Ante esto la Organización Universidad y quienes la conducen deben darle al PASyT las oportunidades, en el contexto de una carrera laboral total e integral, y no una sucesión de cursos, cursillos y cursitos para cubrir puestos de trabajos separados e inconexos.

Urge hoy un Planeamiento Estratégico de Capacitación. Para iniciarlo será menester hacer un diagnóstico adecuado de las fortalezas y debilidades de la organización que se pretende planificar y tener información útil del contexto específico, así como del medio ambiente general, que nos permita identificar oportunidades y amenazas. 
Veremos que es preciso lograr algunos acuerdos mínimos sobre cómo y qué analizaremos de nuestro potencial organizacional y su situación vigente, con qué esquema axiológico mediremos cada recurso, cada proceso y cada resultado y cuáles serán los indicadores (cualitativos o cuantitativos) que emplearemos en el análisis.

Establecer las variables significativas de todo tipo (normativas, sociológicas, económicas, políticas) y sus respectivos indicadores, las fuentes de información a emplear, así como el posible impacto que cada variable tendrá en nuestro desempeño futuro y también, quienes serán los actores significativos.

H. Simón (1964) plantea el tema de los "juicios de valores" y las "racionalidades decisorias". El concepto de lo "bueno" o "deseable" y de lo "malo" y "evitable", dependerá precisamente de estos juicios.

Se deberá acordar acerca de la visión, y sus objetivos estratégicos, recordando que lo meritorio del planeamiento estratégico no es posible evaluarlo a corto plazo, como todo lo referido a la educación, porque las mediciones de eficacia se realizan sobre el impacto que éste imprime a la calidad institucional y en la sociedad que lo recibe.

La mundialización de los sistemas de comunicación nos empuja hacia una realidad, o la universidad adopta las estrategias de planificación, coordinación, control y evaluación en pos de su propia evolución, o dejará de ser. La experiencia nos indica que el problema de la eficiencia del funcionamiento universitario no se puede lograr por mandato, sino desde la capacidad de adaptación a estos cambios y, en definitiva, de la competencia del PASyT, para lo cual se necesitan programas de capacitación especiales, actividades específicas de desarrollo y asesoramiento con el fin de prepararlos para funciones laborales universitarias exitosas. Asimismo para aquellos que ya poseen conocimientos de dirección, será necesario implementar un programa de planeamiento de carrera para maximizar su contribución potencial a la organización universitaria.

Para la conducción gremial, también interesada por todas las implicancias del planeamiento de carrera, ésta es la herramienta que permite adquirir al PASyT los conocimientos que lo ayudarán a soportar los altos valores de cambio organizacional.

Es decir, el propósito de un programa de planeamiento de carrera conlleva a incrementar las posibilidades de que el PASyT alcance sus logros y, a su vez, la Universidad se asegure de contar con un capital humano importante para ubicarlo en el tiempo y lugar de trabajo en el que surja cualquier demanda.

Para compatibilizar los objetivos del PASyT y las necesidades de la organización universidad, ambos se deben definir explícitamente. Se deberán dar a publicidad o conocimiento los proyectos para la creación de nuevas funciones, los cambios en la estructura orgánico-funcional producto de la expansión estratégica de la organización universitaria, ya sea por las nuevas creaciones o por la jerarquización de las funciones que pueden afectar sus carreras significativamente. 
La preparación y desarrollo de los cursos de capacitación y perfeccionamiento deben satisfacer diversas exigencias, como ser mayor y mejor preparación:

- Para actuar en lo referente a la política y administración de los recursos.

- Para coadyuvar a la planificación de la administración universitaria.

- En tecnología de gestión sobre la administración universitaria.

- En legislación laboral de las Universidades.

La diversidad de tareas para cumplir las funciones del PASyT, existentes y a crear en el futuro, son diferentes en el espacio y en el tiempo. Esto hace necesario el desarrollo de programas que deberán servir a nuevos objetivos, tanto de desarrollo técnico como humano. Para este tipo de necesidades se deberán preparar pequeñas experiencias con grupos reducidos y las actividades o programas deberían ser con la modalidad presencial y de educación continua.

Tendremos que saber si la conducción universitaria, en razón de que el diseño y su implementación representan una nueva visión en las relaciones Universidad-Trabajador, está dispuesta a enfrentar el nuevo desafío, ya que requiere de una inversión importante y considerable de tiempo, de capital humano y de recursos económicos, tanto por parte de la Universidad como por parte del trabajador del claustro del PASyT. Una vez aceptada la idea del programa, los responsables de la conducción universitaria, tanto políticos como del PASyT, deberán comprometerse con los objetivos del mismo.

Asimismo, el área encargada o responsable de la implementación del programa de planeamiento de capacitación debe tener un nivel destacado dentro de la estructura orgánico-funcional de la Universidad y estará a cargo de un integrante del PASyT, especialista y con capacitación probada en Gestión de Gobierno de Instituciones de Educación Superior.

Desde un ámbito burocrático eficaz, eficiente y efectivo, y con capacitación permanente, en el que se deben tomar decisiones que permitan aunar el cumplimiento de la ley -teniendo en cuenta el interés de la sociedad en la que está inmersa la universidad- con una gestión eficaz relevante, considero de sumo valor el análisis y la evaluación de los nuevos y renovados conceptos que se hace necesario dominar para lograr avances en una tarea investigativa sobre la gestión de gobierno de las Instituciones de Educación Superior.

¿Vale la pena invertir capital humano, recursos económicos y tiempo en una tarea de esta naturaleza? La respuesta, sin duda, es afirmativa, así nos lo está demostrando la realidad.

Experiencias a nivel internacional, como el caso de las universidades españolas, muestran que las instituciones de educación superior que han adoptado el planeamiento estratégico y la evaluación han logrado mejorar sustantivamente y brindar una formación de calidad (Thorne, 2001). 
Deberíamos aplicar a la gestión universitaria, una construcción integradora que se ocupe simultánea o alternativamente de los aspectos sociológicos y psicológicos del sistema educativo, concibiéndolo como un sistema abierto, en el que puedan trabajar interdisciplinariamente e interrelacionados los claustros que la integran.

Un desafío a lograr será el de desarrollar procedimientos prácticos, desde la perspectiva teórica y metodológica adecuados, con fundamentos científicos, hacia la puesta en marcha de los mismos, con el fin de aplicar las capacidades técnicas y administrativas en el proceso de toma de decisiones, en la institución.

Para que ésta pueda cumplir su Misión hacia la comunidad será necesario mejorar sustantivamente los sistemas de información disponibles y facilitar el mayor acceso a la más amplia transparencia, respecto de los problemas que la aquejan y de las vías que se siguen para resolverlos.

El uso de las Tecnologías de la información y la Comunicación (TICs) han comenzado a producir una sorprendente reingeniería en las comunicaciones de todo tipo y tamaño, abandonando el modelo burocrático-jerárquico para adaptarse al funcionamiento en red, que es una característica de la sociedad de la información.

La adopción de este modelo de organización en el sistema de la Educación Superior es urgente, no sólo para un desempeño ágil y actualizado de las funciones sustantivas de la universidad sino, fundamentalmente, para el ejercicio de un liderazgo inteligente de todos los actores, dentro y fuera de los límites nacionales.

\section{¿Cuál será entonces el perfil deseado del PASyT universitario?}

Los cambios en las nociones de espacio y de tiempo en las sociedades actuales nos llevan a repensar la gestión de las organizaciones universitarias y la cuestión sobre el perfil deseable del profesionista universitario.

Evidentemente el mismo estará ligado a las ideas que podamos tener sobre las funciones históricas de la universidad y los modelos de gestión que consideremos más valederos.

Hoy los desafíos de la gestión universitaria nos conducen a: diseñar sistemas de información inteligentes, que permitan medir y conocer variables significativas, que logren una adecuada socialización de la información neurálgica de la Universidad (Vega, 2005, p. 229).

El conocimiento y uso de las TICs deben integrarse al proyecto pedagógico institucional como parte del planeamiento estratégico, en todos los claustros, con el fin de hacer de la universidad argentina una "organización inteligente".

Ante estos desafíos, los profesionistas, tienden a surgir como individuos muy motivados, dedicados a su trabajo y a quienes atienden o hacia quienes prestan sus servicios. 
Si acordamos que una gestión educativa relevante se evalúa en términos de los significados y las consecuencias de su actuación para el mejoramiento del desarrollo humano y la calidad de vida en la universidad y la sociedad; la percepción e interpretación de esos significados y sus consecuencias sólo serán posibles a través de una teoría organizacional y planificada estratégicamente, construida sobre la base de la experiencia real.

Las exigencias de la administración en plena reconversión y transformación, impone la formación de capital humano que reúna el conocimiento sustantivo del área de gestión con el uso de tecnologías de gestión.

Organismos internacionales, como el Programa de Educación Superior de la OEI (Organización de Estados Iberoamericanos) para el año 2001-2002, han reconocido el papel crucial de la cooperación educativa y cultural para el desarrollo, a través de la movilidad de estudiantes, docentes, investigadores y gestores de la Educación y la importancia de la consolidación de espacios institucionales y sociales con una dimensión iberoamericana.

Esto implica una co-responsabilidad interinstitucional-gubernamental, frente al proceso de internacionalización de la Educación Superior, por lo que prevén entre sus objetivos establecer líneas de acción para el desarrollo de políticas universitarias (hacia adentro de la Organización) y políticas públicas universitarias y científicas (hacia afuera de la Organización).

El capital humano profesionista con el que cuenta nuestra universidad para desarrollar su misión es en general escaso. Esto no justifica la ineficiencia sino por el contrario, se transforma en un desafío requiere de una capacitación permanente que hará que el rendimiento sea mayor y mejor.

\section{Conclusiones}

Para una eficaz acción en la gestión se deben preparar Profesionistas y Profesionales que hagan de su trabajo una vocación de servicio.

Su tarea debe ser fructífera como la del artesano en el arte de combinar capital humano, capacitación específica, infraestructura inadecuada u obsoleta y bajos recursos financieros. Es el accionar de las personas lo que produce el proceso educativo de cambio, no las cosas materiales con las que contamos.

Si no se conoce con qué herramientas se cuenta para trabajar, es imposible que las mismas sean ejecutadas en el accionar diario que torne efectivo y relevante el trabajo.

En los términos del paradigma multidimensional de administración de la educación, la elección y preparación de gestores y profesionistas de la educación debe tener en cuenta cuatro tipos de competencias: económica, pedagógica, política y cultural.

La competencia económica del técnico de la educación define su eficiencia para optimizar la capacitación y utilización de los recursos económicos y 
financieros y de elementos informáticos y materiales para la consecución de los objetivos del sistema educativo.

La competencia pedagógica del técnico de la educación refleja su eficacia para formular objetivos educativos y para diseñar escenarios y medios estratégicos para su consecución.

La competencia política define el talento del técnico de la educación para percibir e interpretar el ambiente externo y su influencia sobre las instituciones educativas y revela la efectividad para adoptar estrategias de acción organizada, para satisfacer las necesidades y demandas sociales y políticas de la comunidad y de su sistema educativo.

Finalmente, la competencia cultural del técnico de la educación revela su capacidad para concebir soluciones y el liderazgo para implementar bajo la óptica de la relevancia, la deducción de una forma cualitativa de vida humana que posibilite la plena realización personal de los participantes del sistema educativo y de la sociedad como un todo.

La preparación de investigadores, administradores, técnicos y profesionistas en función de este conjunto de competencias básicas, se presenta como una necesidad y un desafío para los sistemas educativos de América del Sur. Un desafío, ya que se trata de una inmensa tarea académica, teniendo en cuenta la importancia y la complejidad del tema. Una necesidad, pues cabe a los administradores de la educación una tarea central en la organización y la gestión de las instituciones y sistemas de América Latina (Sander, 1996, p. 68).

Si se apuesta por eficiencia, eficacia y responsabilidad en la toma de decisiones es imprescindible apostar por equipos que sepan realizar su gestión con agilidad, eficacia y responsabilidad.

Hay que pensar en todo un período de cambio de los antiguos administradores, a los nuevos profesionistas de la gestión de gobierno administrativo de la educación.

Cuando los estados son fuertes de verdad, tienen también políticas de Estado de verdad que van más allá de lo que piensa este o aquel sector de la sociedad. En la universidad, ello es también condición necesaria para planificar estratégicamente y darle su valiosa importancia a la implementación de los avances informáticos que posibilitan la gestión del conocimiento y la aplicación de cambios oportunos.

A partir de los resultados de nuestra investigación nuestra propuesta concreta y en virtud que son muy pocas las universidades que a la fecha tienen en marcha una carrera formativa específica consideramos, por lo expuesto, que ésta es una de las demandas prioritarias, a resolver.

Se ha dejado de lado un sistema de evaluación de desempeño y esto es desfavorable, ya que toda acción buena, regular o mala tiene el mismo valor, por lo que debería retomarse esta sana práctica que hasta puede convertirse en una especie de estímulo o incentivo, tan necesario en la actualidad. 
Es necesario crear mecanismos de retención de personal, ya que al no existir estos, significa una pérdida valiosa para la Universidad, porque se utiliza un tiempo importante para capacitar, aunque sea de modo informal, al PASyT, que ya capacitado puede optar por un empleo mejor remunerado, o mejor reconocido.

Al momento de habilitarse el Proyecto FOMEC se dio oportunidad a gran cantidad de docentes a recibir capacitación en el exterior, asegurándole a su retorno un cargo docente dedicación exclusiva, sería motivante lograr que posibilidades similares puedan ser capitalizadas por el PASyT. Cuando existe la posibilidad de realizar algún curso, asistir a un seminario o participar en un congreso, el PASyT, debería estar informado por la vía de la autoridad correspondiente de la Universidad (hoy día los pocos que se enteran lo logran a través de correos personales). La Universidad debería facilitar a sus empleados el acceso a becas, residencias, u otros beneficios, que meriten la profesionalización del PASyT.

En consecuencia vemos que urge realizar una gestión imbuida de los beneficios que otorga no sólo el planeamiento estratégico, sino también la gestión del conocimiento en una sociedad inteligente, a fin de diseñar el mejor logro de capital humano (intangible), la mejor calidad de servicios, y el eficiente, eficaz y efectivo accionar en las políticas operacionales de la Universidad a fin de que las mismas se transformen en relevantes, para la sociedad, la región, el país y el mundo con el que interactúan.

Resultará un aporte beneficioso equilibrar la interacción entre los claustros que integran hoy el Gobierno Universitario, ya que el mayor activo de cualquier organización y por ende de cualquier país es la expresión del conocimiento y la planificación que del mismo se haga con el fin de lograr enmarcar a la universidad dentro del conjunto de "Organizaciones que aprenden" (2).

Las modernas teorías de Liderazgo nos enseñan que para llegar a ser un líder (y esto debería ser el propósito de cada uno del los integrantes del PASyT, acorde a su función y el reconocimiento de los que nos rodean, se basa no ya en el poder del puesto, de la voz, del gesto; sino precisamente en la "autoridad del saber" (Vega, 1996).

"Tomando en cuenta las culturas organizacionales vigentes y los nuevos contextos podemos identificar algunos objetivos fundamentales:

- Fortalecer la capacidad de gestión estratégica y abandonar las prácticas sectoriales y corporativas.

- Jerarquizar las funciones directivas y de gestión apelando en lo posible a la formación específica de postgrados para ello.

- Crear nexos permanentes entre las prácticas de gestión, el diseño de políticas de conocimiento y de información, los cambios y reformas curriculares, los programas de evaluación y las reformas institucionales. 
Tanto la capacitación y el desarrollo del personal, como las bonificaciones e incentivos tiene estrecha relación con uno de los temas más importantes que moviliza a todo ser humano, la motivación" (Padrino, 2005, p. 179).

"Las complejidades que enfrentamos requieren cualidades especiales para situarse en el plano de las políticas de conocimientos, de los desafíos culturales y sociales que han de definir el destino de nuestros pueblos, a los que nos debemos como parte de nuestro compromiso ético e institucional" (Pérez Lindo, 2005, p. 197).

Promover la rotación del personal en las tareas, cuando sea posible, es lo que le permitirá adquirir habilidades diferentes. El nuevo convenio colectivo de trabajo abunda en el requisito, la necesidad y la oportunidad de la capacitación permanente, y la disposición de las partes firmantes a brindarle al trabajador los medios indispensables para lograrlo.

Hoy, más que nunca, la sociedad inteligente y la gestión del conocimiento nos ponen frente al deseo de la capacitación continua. Es casi una obligación, para nosotros, responder a este desafío en pos de una Nación educadamente soberana.

La Gestión Universitaria práctica debe profesionalizarse y adecuarse al contexto de la política nacional de educación. Para preservar nuestra identidad, es necesario que la orientación general de la política educativa sea de índole nacional.

Esta política nacional de educación debe traducirse en un núcleo curricular mínimo que permita salvaguardar la identidad cultural del país, fortalecer su capacidad de construcción del conocimiento científico y brindar igualdad de oportunidades de acceso al desarrollo tecnológico, del cual ya no podemos prescindir si nos consideramos ciudadanos del mundo.

La gestión organizacional del siglo XXI empieza a olvidarse del modelo tradicional de liderazgo basado en una mentalidad conservadora, paternalista, y autoritaria que, cada vez más, está siendo sustituida por una mentalidad amplificadora, con un modelo nuevo, moderno, emprendedor y motivador.

$\mathrm{Si}$ se toma conciencia de los estrechos vínculos que hay entre educación y sociedad, y la gestión se coordina y engloba en las perspectivas de transformación social, económica y política, que la historia está pidiendo a los claustros universitarios en su accionar como actores sociales, comprometidos con su entorno, podemos avizorar un país con futuro, que se orienta hacia la universalización de su Educación Superior, para un mundo mejor. 


\section{Notas Bibliográficas}

(1) Ordoñez de Pablos, P. (s.f.) Medición y elaboración de informes de capital intelectual. Citado por Di Doménico A. y De Bona, G. (2005) "Activos intangibles en Organizaciones de Educación Superior. Medición e Indicadores del Capital Intelectual ", en Aportes al debate sobre la Gestión Universitaria I. Buenos Aires, De los Cuatro Vientos.

(2) Ver Pérez Lindo, A. (Comp.) (2005) La Gestión del Conocimiento. Un nuevo enfoque aplicable a las organizaciones y la Universidad. Buenos Aires, Norma. Y también: Gibbons M. y otros (1997) La nueva producción del conocimiento. Buenos Aires, Pomares Corredor.

(3) La presente es una síntesis actualizada que tiene como base el Libro "La Especificidad de la Gestión Universitaria. Gobierno y administración de su capital humano" y está registrado a nombre de la autora Ana María Trottini. El Libro fue Editado por la Editorial Universidad Nacional de Rosario - Junio de 2007, Argentina.

\section{Referencias Bibliográficas}

- Aguilar Villanueva, L. F. (1996) El estudio de las políticas públicas. México, Miguel Ángel Porrúa.

- $\quad$ Altbach, Ph. G. y Mc Gill Peterson, P. (2000) Educación Superior en el Siglo XXI: Respuesta Nacional. Buenos Aires, Biblos.

Universidad de Palermo. Buenos Aires.

(2001) Educación Superior Comparada.

- Braslavsky, C. y Acosta, F. (2001) El Estado de la Enseñanza de la Formación en Gestión Educativa en América Latina. Buenos Aires, UNESCO IIPE.

- Brunner, J. J. (2002) "Aseguramiento de la Calidad y Nuevas Demandas sobre Educación Superior en América Latina", en Educación Superior, Calidad y Acreditación. Primer Seminario Nacional de Acreditación, 10-12 de Junio de 2002, Cartagena, Colombia.

- FLACSO - FCF.

(1990) Educación Superior en América Latina. Santiago de Chile

- $\quad$ Camilloni, A. R. W. de (2001) "Complejidad Superior: El concepto de Universidad", en Encrucijadas, N 12, U.B.A., Año 1, Octubre, Buenos Aires.

- Carvalho, J. R. (2008) "La premisa fundamental del MESALC es la cooperación regional como el camino más eficiente y eficaz para superar nuestras deficiencias". El Mapa de la Educación Superior en América Latina y el Caribe - MESALC.

- $\quad$ Clark, B. (1991) El Sistema de Educación Superior - Una visión comparativa de la Organización Académica. México, Nueva Imagen/UNAM.

- Castells, M. (2001) Internet y la sociedad en red (síntesis), en Lección inaugural del programa de doctorado sobre la sociedad de la información y el conocimiento. Barcelona, UOC. 
- Etkin, J. (2000) Política, Gobierno y Gerencia de las Organizaciones. Buenos Aires, Prentice Hall.

- Efrón, M. y Pérez Lindo, A. (Comp.) (2005) Aportes al debate sobre la gestión Universitaria I, Buenos Aires, De Los Cuatro Vientos.

- Efrón, M. y Vega, R. I. (Comp.) (2005) Aportes al debate sobre la gestión Universitaria II. Buenos Aires, De Los Cuatro Vientos.

- Fernandez Lamarra, N. (2002) La educación Superior en la Argentina. IESALCUNESCO. Borrador, Buenos Aires.

\section{Buenos Aires, Eudeba.}

(2002) La educación Superior en la Argentina en debate.

- $\quad$ Frigerio, G. y Poggi, M. (1997) El Análisis de la institución educativa. Hilos para tejer proyectos. Buenos Aires, Aula XXI/Santillanas.

mentos para la gestión. Buenos Aires, Troquel.

- García de Fanelli, A.M. (1998) Gestión de las Universidades Públicas: La experiencia internacional. Buenos Aires, Serie Nuevas Tendencias, Ministerio de Cultura y Educación, Secretaría de Políticas Universitarias.

- Gaviria, C. (1995) Discurso de la inauguración de la XXV Sesión ordinaria de la Asamblea General la Organización de los Estados Americanos (OEA), Haití.

- Gibbons, M. y otros (1997) La nueva Producción del Conocimiento. Barcelona, Pomares-Corredor.

XXI. Conferencia Mundlats de octubre. París.

- González, M. L. (1995) “El presupuesto en la administración de la organización universitaria", en Aportes al debate sobre la gestión Universitaria.

- Habermas, J. (1986) Historia y crítica de la opinión pública. La transformación estructural de la opinión pública. México, Gili.

Sociológica, $\mathrm{N}^{\circ} 5$. México, Univ. Autónoma Metropolitana.

- Levy, D.C. (1995) La Educación Superior y el Estado en Latinoamérica. Desafíos privados al predominio público. Temas y conceptos. México, UNAM, CESU.

- Martinez Nogueira, R. (2000) La evaluación de la gestión universitaria, CONEAU, www.coneau.edu.ar/que_es/document/publicaciones/Evaluacion_ gestion_universitaria.PDF

(2000) "La gestión pública: del diálogo crítico a la evaluación de la diversidad”, en Revista Política y Gestión N 1. Rosario, Homo Sapiens.

las instituciones: Cuestiones y aprendizajes. 
(2002) La gestión Universitaria: desafíos para las disciplinas organizacionales y administrativas. www.econo.unlp.edu.ar/dptos/ revistaecos_articulos.htm.

(2003) La evaluación para el análisis y la planificación de las organizaciones. www.administracion.econo.unlp.edu.ar/.../01_principal/01_ principal_c_2004/U11_MARTINEZ\%20NOGUEIRA.pdf.

- Mintzberg, H. (1997a) Diseño de organizaciones eficientes. La Burocracia Profesional. Buenos Aires, El Ateneo.

(1997b) Planeamiento estratégico, La ideología y la Organización visionaria. Buenos Aires, El Ateneo.

- Navarro, A.M. (2000) La evaluación de la Gestión CONEAU Buenos Aires. www.unr.edu.ar/institucional/autoevaluacion.

- $\quad$ Neave, G. y Van Vught, F. (1994) Prometeo encadenado. Estado y educación en Europa.Barcelona, Gedisa.

- Neave, G. (2001) “Universidad y Comunidad ¿Relaciones peligrosas?”, en Encrucijada, N 12, U.B.A., Año 1, Oct. Buenos Aires.

- Nonaka, I. y Takeuchi, H. (1999) La organización creadora de conocimiento: Cómo las compañías japonesas crean la dinámica de la innovación. México, Oxford Press.

- North, D. C. (1995) Instituciones, cambio institucional y desempeño económico. México, F.C.E.

- Ordoñez de Pablos, P. Medición y Elaboración de Informes de Capital Intelectual, citado por Di Domenico http:///www.gestiondelconocimiento.com/ documentos2/patricia/informes.htm.

- Pérez Lindo, A. (1993) Teoría y educación superior. Instituto de estudios y acción social. Buenos Aires.

deba.

(1995) Universidad, Política y Sociedad. Buenos Aires, Eu-

Buenos Aires, Biblos.

- Pérez Lindo, A., Camos, C., Darin, S., Trottini, A.M. y otros (2005) Gestión del Conocimiento. Un nuevo enfoque aplicable a las organizaciones y la universidad. Buenos Aires, Norma.

- Porter, L. (1992) Manual para la Elaboración de un Plan Estratégico. Maestría en Planeación y Desarrollo de la educación. México, UAM-Xochimilco.

- Sander, B. (1990) Educación, administración y calidad de vida. Buenos Aires, Santillana.

(1996) Gestión Educativa en América Latina. Construcción y reconstrucción del conocimiento. Buenos Aires, Troquel.

- Thorne, C. (2001) "La calidad de la educación universitaria y el caso peruano", en La universidad que Perú necesita. Foro Educativo y Consorcio de Universidades, Lima. 
- Trottini, A.M. (2002) La burocracia administrativa y su ingerencia en el apoyo a la gestión de gobierno de la Institución Universidad Pública. Seminario Maestría - UNR - Unigestiones - Publicado en www.riurhc.unc.edu.ar.

- _ (2004) El EGABE (Equipo de Gestión Administrativa Burocrática Eficaz), Trabajo Final de Postgrado Unigestiones2000, UNR/UNL/UNER., en C.D. Congreso de la RIURRHH, Univ. Nac. de Mar del Plata.

- Análisis sobre la estructura organizativa y la capacidad de gestión la Facultad de Ciencia Política y Relaciones Internacionales de la Universidad Nacional de Rosario. (Basado en la Descripción de la Estructura Organizativa, la Elaboración de la Matriz de Producción Externa y la Evaluación de la Capacidad Institucional en www.aaeap.org.ar/ponencias/congreso2/TrottiniDemarco-Burke.pdf).

(2004) La especificidad de la Gestión Universitaria: Gobierno y Administración, en CICSO Revista del Centro de Investigaciones en Ciencias Sociales $N^{\circ}$ 2. Rosario, UNR - Facultad de Derecho. (2007) La especificidad de la Gestión Universitaria. Gobierno y Administración de su Capital Humano. Rosario, U.N.R. Editora, Colección Académica.

- Tunnemann, C. (1996) La educación superior en el umbral del Siglo XXI. Caracas, UNESCO-CRESALC.

- Vega, R. I. (1998) “La Universidad Argentina: ¿una Institución en Crisis?”, en FASES N ${ }^{\circ} 2$ Revista de la Facultad de Cs. Económicas y Ss. Mar del Plata, UNMDP.

$\cdot$

(2004) "El Planeamiento Estratégico en la Universidad Argentina: Aspectos Problemáticos", en Colossi, N. Dias de Souza Pinto (Comp.): Estudos e Perspectivas em Gestâo Universitaria. Blumenau, Nova Letra.

- Viedma Martí, J.M. (2002) La gestión del conocimiento y el Capital Intelectual http://www.gestiondelconocmiento.com/documentos2/viedma/gdcv.htm.

\section{Documentos:}

- Ley 24.521 / Decreto 176/96 / Decreto 2213/87 / Decreto 366/06 "Escalafón para el Personal no docente de las Universidades Nacionales".

- Boletín oficial del Estado.

- Programa de las Naciones Unidas para el desarrollo (PNUD), Informe sobre desarrollo humano, México DF, F. C. E. 1994.

- CONEAU, Lineamientos para la evaluación institucional, Buenos Aires, 1997. 